Research Paper

\title{
Clinical and predictive significance of Plasma Fibrinogen Concentrations combined Monocyte-lymphocyte ratio in patients with Diabetic Retinopathy
}

\author{
Qinghua Huang ${ }^{1,2}$, Hui $\mathrm{Wu}^{1,2}$, Mingyi $\mathrm{Wo}^{3}$, Jiangbo Ma ${ }^{1,2}$, Yingxiang Song ${ }^{1,2}$, Xianming Fei ${ }^{3} \bowtie$ \\ 1. Department of Endocrinology, Zhejiang Provincial People's Hospital, and People's Hospital of Hangzhou Medical College, Hangzhou, Zhejiang, China. \\ 2. Key Laboratory of Endocrine Gland Diseases of Zhejiang Province, Hangzhou, Zhejiang, China. \\ 3. Center for Laboratory Medicine, Zhejiang Provincial People's Hospital, and People's Hospital of Hangzhou Medical College, Hangzhou, Zhejiang, China. \\ $\triangle$ Corresponding author: Xianming Fei, E-mail: feixianming@hmc.edu.cn; Tel.: 86-571-85893171; Address: No. 158 Shangtang Road, Xiacheng District, \\ Hangzhou, Zhejiang, China; Postal code: 310014.
}

(C) The author(s). This is an open access article distributed under the terms of the Creative Commons Attribution License (https://creativecommons.org/licenses/by/4.0/). See http:/ /ivyspring.com/terms for full terms and conditions.

Received: 2020.08.04; Accepted: 2021.01.04; Published: 2021.01.26

\begin{abstract}
Diabetic retinopathy (DR) is one of the most common causes of blindness and visual impairment. Therefore, early prediction of its occurrence and progression is important. This study aimed to assess the clinical and predictive significance of plasma fibrinogen concentrations combined monocyte-lymphocyte ratio (FC-MLR) in patients with DR. A total of 307 patients with type 2 diabetes (T2D) were enrolled. Plasma fibrinogen concentrations and peripheral white blood cells were measured, and MLR was calculated, and the associations of FC-MLR with DR and severity of disease were assessed. Regression analysis and receiver operating characteristic (ROC) curves were performed to evaluate the risk factors and predictive power of FC-MLR for $D R$ and severity of disease, respectively. DR patients showed higher fibrinogen concentrations and a higher MLR than did T2D patients without complications $(P<0.01)$; Moreover, $D R$ patients in proliferative stage also showed higher fibrinogen concentrations and a higher MLR than did those in non-proliferative stage $(P<0.01)$. FC-MLR was closely associated with occurrence and severity of $D R(P<0.01)$, and was an independent risk factor for them $(O R=6.123,95 \% \mathrm{Cl}: 3.122-17.102$; and $7.932,95 \% \mathrm{Cl}: 4.315-16.671$, respectively; $\mathrm{P}<0.001)$. The predictive sensitivity and specificity for DR and severity of disease were 0.86 and 0.68 , and 0.85 and 0.73 , respectively. The study suggests that FC-MLR may be used as a predictor for the risk and progression of diabetic retinopathy.
\end{abstract}

Key words: diabetic retinopathy; fibrinogen concentrations; moncyte-lymphocyte ratio; predictor

\section{Introduction}

AS one of the most common causes, diabetic retinopathy (DR) contributes to $2.6 \%$ and $1.9 \%$ of blindness and visual impairment in worldwide [1]. However, yearly examination of the retina or retinal photography is often delayed until after severe damage even visual impairment has occurred [2]. Therefore, early identification of the microvascular complications risk provides an opportunity to stop or delay disease onset [3], which would be more valuable to improve the prognosis of T2D patients. Studies have shown that many biomarkers can reflect the presence of microvascular damage $[4,5]$, and they are also associated with the risk of retinopathy $[6,7,8]$. However, to our knowledge, most of those markers are not the convenient and practical ones for the prediction, diagnosis, and prognostic assessment of DR. Thus, there are some clinical limitations for them, and it would be of great clinical significance to explore some more valuable markers for early screening and assessing the disease progression of patients with DR.

Increasing evidence points to inflammation as a critical contributor to the development of DR [9], and many inflammatory markers are associated with increased risk of DR [10]. Therefore, inflammationrelated markers would play important roles in prediction and disease assessment of DR. Liu et al. has reported that WBC subtypes were closely associated with inflammatory state of DR patients [11], which indicates that peripheral blood parameters of patients 
would be of great value for DR diagnosis and treatment. Monocyte-lymphocytes ratio (MLR), a novel inflammatory marker, plays an important role in prediction and prognosis of some tumors and cardiovascular diseases $[12,13]$. It has been recognized that tumors and cardiovascular diseases as well as DR are inflammation-related diseases. However, the associations of MLR with DR remains unclear. On the other hand, fibrinogen is an acute-phase reactant glycoprotein mainly synthesized by hepatocytes [14]. Furthermore, plasma fibrinogen is important in the regulation of inflammatory response [15], and plasma fibrinogen levels are associated with increased inflammation [10]. Our previous studies have revealed that plasma fibrinogen is a powerful predictor for diabetic nephropathy in patients with T2D [16], and combined plasma fibrinogen concentrations and MLR (FC-MLR) can be used as a powerful prognostic index of ovarian cancer [18]. Therefore, fibrinogen may play a combined role with MLR in inflammatory diseases. Although the associations of FC-MLR with DR is unknown, we speculated that both plasma fibrinogen concentrations and MLR may play important roles in development and progression of DR, and there would be a valuable significance of combined FC-MLR in DR patients. However, the association of FC-MLR with DR is unknown. Thus, the aim of this study was to explore the clinical and predictive significance of FC-MLR in DR patients.

\section{Materials and methods}

\section{Patients' population}

A total of 307 patients with T2D were finally enrolled in the study. All patients were the outpatients or inpatients from the Departments of Endocrinology, Zhejiang Provincial People's Hospital, China, between October 2015 and December 2016. T2D patients without diabetes-related complications and those with retinopathy only before daily treatment were included in the study. All patients with T2D were diagnosed according to the criteria in the 2013 Diabetes Guideline from the China Diabetes Association [18]. Patients who exhibited the following characteristics were included: 1) typical symptoms of diabetes (polyuria, polydipsia, unexplained weight loss); 2) random blood glucose level $\geq 11.1 \mathrm{mmol} / \mathrm{l} ; 3$ ) fasting plasma glucose (FPG) level $\geq 7.0 \mathrm{mmol} / 1$ or 2 -h post-challenge glucose level in oral glucose tolerance test $\geq 11.1 \mathrm{mmol} / 1$. Any atypical symptoms were confirmed on a different day. The following exclusion criteria was used: 1) diabetes-related complications except DR; 2) primary liver and kidney dysfunctions; 3) cardiovascular and cerebrovascular diseases; 4) malignancies; 5) acute inflammation or infections; 6) postoperative status; and 7) incomplete data. Retinopathy of diabetic patient was assessed using digital retinal photographs. According to severity of disease, DR is divided into two stages: nonproliferative diabetic retinopathy (NPDR, an early stage) and proliferative diabetic retinopathy (PDR, an advanced stage). NPDR and PDR are characterized as follows, respectively: during NPDR stage, retinal pathologies including microaneurysms, hemorrhages and hard exudates can be detected by fundus photograph, and during PDR stage, the patients may experience severe vision impairment when the new abnormal vessels bleed into the vitreous (vitreous hemorrhage) or when tractional retinal detachment is present [19]. The basic evaluation before daily diabetic-related treatment included clinical assessment (disease course: time since diagnosis of diabetes, risk factors, data of physical examination, and clinical and biologic data), and the major laboratory tests. T2D patients were divided into two groups including T2D without diabetic-related complications group (212 patients) and T2D with retinopathy group (95 patients). At the same time, 101 healthy controls (age, sex, and race-matched) were included in the study. The control subjects came from the healthy management center at the hospital, and had not taken any drugs in the 2 weeks before sample collection. Informed consent was obtained from the controls and patients, and this study was approved by the Hospital's Ethics Committee.

\section{Laboratory assay}

Samples were collected and prepared as the description previously [20, 21]. In brief, venous blood samples were collected in sodium citrate- or EDTA$\mathrm{K}_{2}$-containing and anticoagulant-free vaccutainer tubes (Becton Dickinson, MountainView, CA, USA) after an overnight fast before treatment, respectively. Prothrombin time (PT), international normalized rario (INR), activated partial thromboplstin time (aPTT), thrombin time (TT), fibrinogen (Fbg), and D-dimer (D-D), were measured by a coagulation analyzer (CS-5100, Japan Sysmex) and the commercial reagents (Siemens Healthcare Diagnostics Products $\mathrm{GmbH}$ ) within four hours after sample collection, respectively. Total WBC, monocyte, and lymphocyte levels were measured by an automatic hematological analyzer and commercially available reagents (BC-6900; Mindray Inc., Shenzhen, China) in one hour after sample collection. Sera were obtained from the blood samples without anticoagulants by centrifugation at $1500 \times \mathrm{g}$ for $10 \mathrm{~min}$ at room temperature. Subsequently, the levels of other biochemical indexes including plasma high-sensitive 
C-reactive protein, serum Cystatin C, Thyroid stimulating hormone, Fasting serum glucose, Peptide-C, Glycosylated hemoglobin, Serum high density lipoprotein, Total cholesterol, and Albumin were measured. At the same time, the biological and clinical data of the patients and controls (age, sex, and body mass index for all subjects; T2D-related and pathological data before in-hospital treatment for all patients) were collected and reviewed. The analyzers were well calibrated, and an internal quality analysis was well performed. All experiments were performed in accordance with relevant guidelines and regulations.

\section{Statistical analysis}

Data were initially tested for distribution normality by the Kolmogorov-Smirnov test, and normally distributed data were presented as mean \pm standard deviation. One-way analysis of variance was used to analyze the differences among among controls, T2D and DR patients, and differences between two groups were subsequently analyzed by the Student-Newman-Keuls (SNK) test. Samples with normal and non-normal data distributions were analyzed by Student's $t$-test and the Mann-Whitney $U$-test, respectively. The Chi-square test was used for categorical variables (clinical and biological characteristics and patient percent in different FC-MLR groups). Multivariate logistic regression analyses were performed to calculate the odds ratio and $95 \%$ confidence interval $(95 \% \mathrm{CI})$ for DR. A receiver-operating characteristic (ROC) curve was constructed, and the area under the curve (AUC) was calculated to evaluate the predictive power of the independent risk factors. All statistical analyses were performed using the SPSS 20.0 statistical package (SPSS, Chicago IL, USA). A value of $P<0.05$ was considered statistically significant.

\section{Results}

\section{Clinical and biological characteristics of patients with type 2 diabetes}

In this study, 307 consecutive patients with confirmed diagnosis of T2D were enrolled. The present study initially investigated the basic characteristics of the patients. The median age at diagnosis was 62 years old (range: $37-85$ to 85 years), and male patients constituted majority of the group $(n=201,65 \%)$. Overall, about $65 \%$ of the patients showed elevated plasma fibrinogen concentrations and MLR $(P<0.05)$. The detailed comparisons of the basic clinical and biological characteristics are presented in Table 1.
Table 1. Clinical and biological characteristics of patients with type 2 diabetes

\begin{tabular}{|c|c|c|c|}
\hline & Number & $\%$ & $P$ value \\
\hline $\mathrm{n}$ & 307 & 100 & \\
\hline \multicolumn{4}{|l|}{ Age, years } \\
\hline Median (range) & $62(37-85)$ & & \\
\hline-60 & 160 & 52 & \\
\hline $60+$ & 147 & 48 & $>0.05$ \\
\hline \multicolumn{4}{|l|}{ Gender } \\
\hline Male & 201 & 65 & \\
\hline Female & 106 & 35 & $<0.05$ \\
\hline \multicolumn{4}{|l|}{ Complications } \\
\hline Without & 212 & 69 & \\
\hline Retinopathy & 95 & 31 & $<0.01$ \\
\hline \multicolumn{4}{|l|}{ DR Stage } \\
\hline Non-proliferative stage & 34 & 36 & \\
\hline Proliferative stage & 61 & 64 & $<0.01$ \\
\hline \multicolumn{4}{|l|}{ Systolic pressure } \\
\hline$\geq 140 \mathrm{mmHg}$ & 60 & 20 & \\
\hline$<140 \mathrm{mmHg}$ & 247 & 80 & $<0.01$ \\
\hline \multicolumn{4}{|l|}{ eGFR } \\
\hline$\geq 90 \mathrm{ml} / \mathrm{min}$ & 234 & 76 & \\
\hline$<90 \mathrm{ml} / \mathrm{min}$ & 73 & 24 & $<0.01$ \\
\hline \multicolumn{4}{|l|}{ UAE } \\
\hline$\geq 300 \mathrm{mg} / 24 \mathrm{~h}$ & 104 & 34 & \\
\hline$<300 \mathrm{mg} / 24 \mathrm{~h}$ & 203 & 66 & $<0.05$ \\
\hline \multicolumn{4}{|c|}{ Thyroid stimulating hormone } \\
\hline Normal & 210 & 68 & \\
\hline Elevated & 97 & 32 & $<0.01$ \\
\hline \multicolumn{4}{|l|}{ Fasting serum glucose } \\
\hline Normal & 43 & 14 & \\
\hline Elevated & 264 & 86 & $<0.01$ \\
\hline \multicolumn{4}{|l|}{ Peptide-C } \\
\hline Normal & 181 & 59 & \\
\hline Elevated & 126 & 41 & $>0.05$ \\
\hline \multicolumn{4}{|c|}{ Glycosylated hemoglobin A1c } \\
\hline$<7.0 \%$ & 234 & 76 & \\
\hline$\geq 7.0 \%$ & 73 & 34 & $<0.01$ \\
\hline \multicolumn{4}{|c|}{ Serum high density lipoprotein level } \\
\hline Normal & 174 & 57 & \\
\hline Decreased & 133 & 43 & $>0.05$ \\
\hline \multicolumn{4}{|l|}{ Serum cholesterol } \\
\hline Normal & 199 & 65 & \\
\hline Elevated & 108 & 35 & $<0.05$ \\
\hline \multicolumn{4}{|l|}{ Serum albumin } \\
\hline Normal & 256 & 83 & \\
\hline Decreased & 51 & 17 & $<0.01$ \\
\hline \multicolumn{4}{|l|}{ Plasma fibrinogen } \\
\hline Normal & 201 & 65 & \\
\hline Elevated & 106 & 35 & $<0.05$ \\
\hline \multicolumn{4}{|l|}{ MLR } \\
\hline Normal & 204 & 66 & \\
\hline Elevated & 103 & 34 & $<0.05$ \\
\hline
\end{tabular}

t. Normal, elevated or decreased criteria was based on the upper or lower limit of reference interval of variables, respectively. eGFR: estimated glomerular filtration rate; UAE: urinary albumin excretion; MLR: monocyte-lymphocyte ratio; $P$ value: comparisons of patient percentage between the two groups by Chi-square test.

\section{Clinical and biological parameters of T2D patients and controls}

Furthermore, the study also assessed the differences between the basic clinical and biological parameter levels among the control subjects and the patients. There were increases in D-D, CRP, and WBC subtypes levels except for a decreased lymphocyte count in T2D patients without complications $(\mathrm{P}<0.01)$. 
However, DR patients showed significantly higher levels of D-D, CRP, and monocyte counts, but a decreasing of lymphocyte and PLT count than did the patients without complications $(\mathrm{P}<0.01$ for all variables). Other parameters did not exhibit significant differences between the groups. The detailed data were presented in Table 2.

Table 2. Comparisons of clinical and biological parameters of $\mathrm{T} 2 \mathrm{D}$ patients and controls

\begin{tabular}{|c|c|c|c|c|}
\hline Variables & Controls & $\begin{array}{l}\text { T2D patients } \\
\text { without } \\
\text { complications }\end{array}$ & DR patients & $P$ \\
\hline $\mathrm{n}$ & 101 & 212 & 95 & \\
\hline Gender (M/F) & $68 / 33$ & $129 / 83$ & $60 / 35$ & $>0.05$ \\
\hline Age, years ${ }^{\Delta}$ & $56(33-79)$ & $57(31-82)$ & $67(35-87)$ & $>0.05$ \\
\hline PT, s & $11.3 \pm 1.4$ & $11.0 \pm 1.5$ & $11.2 \pm 1.6$ & $>0.05$ \\
\hline INR & $0.98 \pm 0.3$ & $0.95 \pm 0.2$ & $0.96 \pm 0.4$ & $>0.05$ \\
\hline $\mathrm{aPTT}, \mathrm{s}$ & $26.5 \pm 3.8$ & $25.2 \pm 3.2$ & $26.0 \pm 4.7$ & $>0.05$ \\
\hline PLT, $10^{9} / \mathrm{L}$ & $211(89-379)$ & $201(55-477)$ & $189(75-664)^{* *}$ & $<0.01$ \\
\hline $\mathrm{D}-\mathrm{D}, \mathrm{mg} / \mathrm{L}$ & $0.20(0.05 \sim 0.48)$ & $0.44(0.05-4.01)^{*}$ & $0.53(0.06-6.31)^{* *}$ & $<0.01$ \\
\hline TT, s & $17.6 \pm 1.4$ & $16.8 \pm 1.3$ & $17.4 \pm 1.2$ & $>0.05$ \\
\hline WBC $\left(\times 10^{9} / \mathrm{L}\right)$ & $5.81 \pm 1.65$ & $7.15 \pm 1.88^{*}$ & $6.95 \pm 2.11^{*}$ & $<0.01$ \\
\hline CRP (mg/L) & $1.25(0.20 \sim 6.34)$ & $4.55(0.20 \sim 15.41)^{*}$ & $8.91(0.20 \sim 23.22)^{\star * *}$ & $<0.01$ \\
\hline Neutrophil $\left(\times 10^{9} / \mathrm{L}\right)$ & $3.78 \pm 1.03$ & $4.55 \pm 1.22^{*}$ & $4.53 \pm 1.25^{*}$ & $<0.01$ \\
\hline Monocyte $\left(\times 10^{9} / \mathrm{L}\right)$ & $0.30 \pm 0.09$ & $0.50 \pm 0.10^{*}$ & $0.66 \pm 0.12^{* *}$ & $<0.01$ \\
\hline Lymphocyte $\left(\times 10^{9} / \mathrm{L}\right)$ & $1.45 \pm 0.21$ & $1.85 \pm 0.30^{*}$ & $1.65 \pm 0.24^{\star *}$ & $<0.01$ \\
\hline
\end{tabular}

†. Data were presented as mean and standard deviation, and the variables with $\left.{ }^{(}\right)$ were present as median and the range. T2D: type 2 diabetes mellitus; DR:diabetic retinopathy; M: males; F: females; PT: prothrombin time; INR: international normalized ratio; aPTT: activated partial thromboplastin time; PLT: platelet count D-D: D-dimer; TT: thrombin time; WBC: white blood cell; CRP: C-reactive protein. $P$ value: comparisons of the three groups by one way analysis of variance. ${ }^{*} P<0.01$ : compared with Controls, and ${ }^{* *} P<0.001$ : compared with T2D without complications analyzed by SNK-test.

\section{Associations of plasma fibrinogen concentrations with clinical characteristics of T2D patients}

In this study, the associations of plasma fibrinogen concentrations with the clinical characteristics of T2D patients were assessed. The mean plasma fibrinogen concentrations were $3.29 \pm 1.00$ and $2.60 \pm 0.63 \mathrm{~g} / \mathrm{L}$ in patients with DR and without complications, respectively. Plasma fibrinogen concentrations were significantly associated with the occurrence of DR $(P<0.01$, Figure 1A). Further study showed that plasma fibrinogen concentrations were significantly higher in patients with proliferative stage than those with non-proliferative stage $(3.49 \pm 0.77 \mathrm{~g} / \mathrm{L}$ vs. $2.99 \pm 0.63$ $\mathrm{g} / \mathrm{L}, \mathrm{P}<0.001$; Figure 1C). The ROC curve analysis indicated plasma fibrinogen had a high AUC [AUC: 0.835; 95\% confidence interval (CI): 0.775-0.896; $\mathrm{P}<0.01]$ for discriminating between patients with $\mathrm{DR}$ and T2D without complications. When the optimal cutoff value was set at $2.95 \mathrm{~g} / \mathrm{L}$, the sensitivity and specificity were 0.78 and 0.71 , respectively (Figure 1B). Moreever, the ROC curve also demonstrated a high AUC (AUC: 0.778; 95\% CI: 0.729-0.828; P<0.01) of plasma fibrinogen concentrations, with an optimal cutoff value of $3.23 \mathrm{~g} / \mathrm{L}$ for discriminating between the DR patients with proliferative and non-proliferative stage. And based on that cutoff value, the sensitivity and specificity were 0.82 and 0.75, respectively (Figure 1D). For the subsequent study, patients were assigned to normal and high fibrinogen groups according to the cutoff values.

\section{Associations of the MLR with the clinical characteristics of T2D patients}

We further investigated the associations of MLR with the clinical characteristics of T2D patients. In patients with DR, MLR was significantly higher than that in patients with $\mathrm{T} 2 \mathrm{D}$ without complications $(0.40 \pm 0.11$ vs. $0.27 \pm 0.08, \quad \mathrm{P}<0.01$; Figure 2A). Furthermore, MLR in DR patients with proliferative stage was remarkably higher than that in patients with non-proliferative stage $(0.430 \pm 0.12$ vs. $0.355 \pm 0.11, \mathrm{P}<0.01$; Figure 2C). The ROC curve analysis showed a high AUC (AUC: $0.868 ; 95 \% \mathrm{CI}$ : 0.823-0.912; $\mathrm{P}<0.01)$ of MLR for discriminating between the patients with DR and T2D without complications. And the sensitivity and specificity were 0.81 and 0.71 , respectively, when the optimal cutoff value was set at 0.26 (Figure 2B). At the same time, we also found MLR had a high AUC (AUC: 0.820; 95\% CI: 0.753-0.8886; $\mathrm{P}<0.01$ ) with an optimal cutoff value of 0.30 for discriminating between the DR patients with proliferative and non-proliferative stage, and the sensitivity and specificity were 0.85 and 0.73, respectively (Figure 2D). For the following study, patients were divided to two groups including a normal and a high MLR according to the cutoff values.

\section{Associations of FC-MLR with DR and disease condition}

Given that plasma fibrinogen concentrations and MLR were closely associated with the occurrence and severity of DR, we simultaneously investigated the associations of plasma fibrinogen concentrations combined MLR with DR and disease condition. We first divided the patients into three groups (group FC-MLR 1: no abnormality; group FC-MLR 2: either high fibrinogen concentrations or a high MLR; group FC-MLR 3: both high plasma fibrinogen concentrations and a high MLR) according to their cutoff values. We found that there was a significantly higher percent of DR patient than that of T2D patients without complications in group FC-MLR 3, and the percent of DR patient in group FC-MLR 1 was significantly lower than that in group FC-MLR 3 (57.9\% vs. $11.4 \%$ and $10.5 \%$ vs. $66.0 \%$, respectively, both $\mathrm{P}<0.01$; Figure 3A). Moreover, there was a gradual increasing of percent of the DR patient with 
proliferative stage from group FC-MLR 1 to 3 (11.1\%, $64.7 \%$, and $84.6 \%$, respectively, $\mathrm{P}<0.01$; Figure $3 \mathrm{C}$ ). The ROC curve analysis further showed a high AUC (AUC: 0.895; 95\%CI: 0.848-0.942; P<0.01) of FC-MLR for discriminating between the patients with DR and T2D without complications, and the sensitivity and specificity were 0.86 and 0.68 based on the optimal cutoff values of fibrinogen and MLR, respectively (Figure 3B). At the same time, the study also exhibited a high AUC (AUC: 0.852; 95\% CI: 0.813-0.889; P<0.01) of FC-MLR for discriminating between DR patients with proliferative and non-proliferative stage, which the sensitivity and specificity were 0.85 and 0.73 according to the cutoff values of fibrinogen and MLR, respectively (Figure 3D).

\section{Associations of plasma fibrinogen, the MLR, and FC-MLR with the risk and progression of DR}

We finally investigated the associations of plasma fibrinogen, MLR, and FC-MLR as well as $\mathrm{HbA1c}$ and disease course, recognized risk factors for DR, with the risk and progression of DR. For this purpose, a multivariate analysis was performed. The analysis demonstrated that plasma Fbg concentrations, MLR and FC-MLR were independently correlated with the risk and progression of $\mathrm{DR}$, and all were the independent predictors for them $(\mathrm{P}<0.001$, respectively). The detailed data were presented in Table 3.

\section{Discussion}

The present study measured plasma Fbg concentrations and MLR in 307 patients with T2D. We also assessed the associations of Fbg, MLR and FC-MLR with DR and its severity. Within our knowledge, this might be the first study to investigate the clinical significance of FC-MLR in patients with DR. Two of the major findings in the study were that FC-MLR was significantly correlated with risk and severity of DR, and it might be used as a predictor for the occurrence and progression of DR.

Table 3. Multivariate Logistic regression analysis in T2D and DR patients

\begin{tabular}{lllllll}
\hline Variables & \multicolumn{2}{l}{ For risk of DR } & \multicolumn{4}{l}{ For progression of DR } \\
\cline { 2 - 7 } & OR & $95 \% \mathrm{CI}$ & $P$ & OR & $95 \% \mathrm{CI}$ & $P$ \\
\hline Course of disease & 13.771 & $7.425-22.893$ & 0.000 & 16.012 & $8.645-33.778$ & 0.000 \\
Fbg & 4.516 & $2.886-13.655$ & 0.000 & 4.289 & $2.401-10.430$ & 0.000 \\
MLR & 5.302 & $2.925-15.201$ & 0.000 & 6.272 & $3.689-15.312$ & 0.000 \\
FC-MLR & 6.123 & $3.122-17.102$ & 0.000 & 7.932 & $4.315-16.671$ & 0.000
\end{tabular}

†. T2D: type 2 diabetes; DR: diabetic retinopathy; Fbg: fibrinogen; MLR: monocyte-lymphocyte ratio; FC-MLR: fibrinogen concentrations combined the monocyte-lymphocyte ratio; OR: odds ratio. CI: confidence interval.
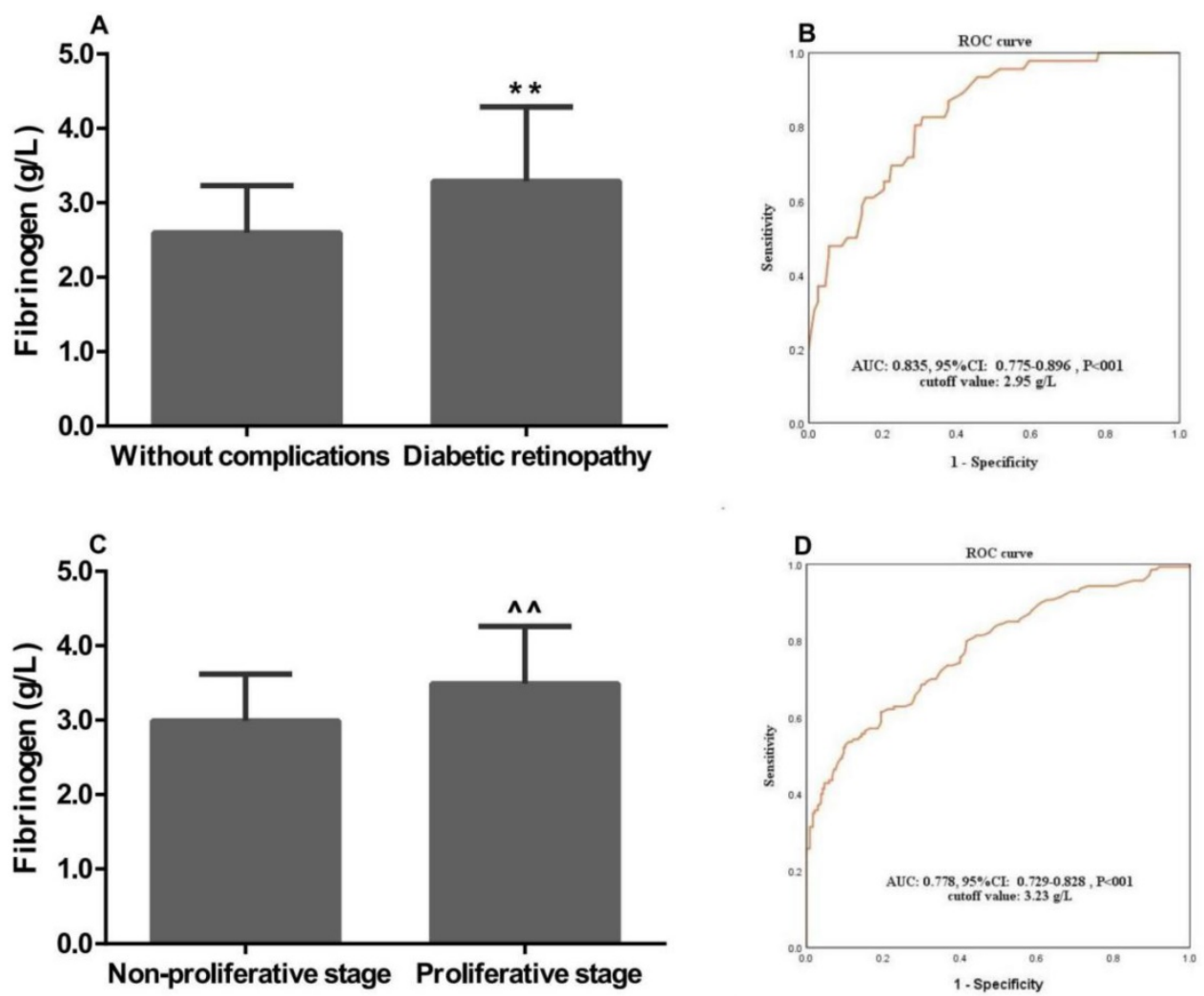

Figure 1. Associations of plasma fibrinogen concentrations with the clinical characteristics of patients with T2D and DR. Fibrinogen concentrations was significantly associated with (A) diabetic retinopathy, and (C) proliferative stage; ROC curve for discriminating between patients with (B) DR and T2D without complications, and (D) non-proliferative and proliferative stage; ROC: Receiver-operating characteristic; T2D: type 2 diabetes; DR: diabetic retinopathy; AUC: area under the curve. **, ^^, P<0.01 vs. "Without complications" and "Non-proliferative stage" patients by the $t$-test, respectively. 

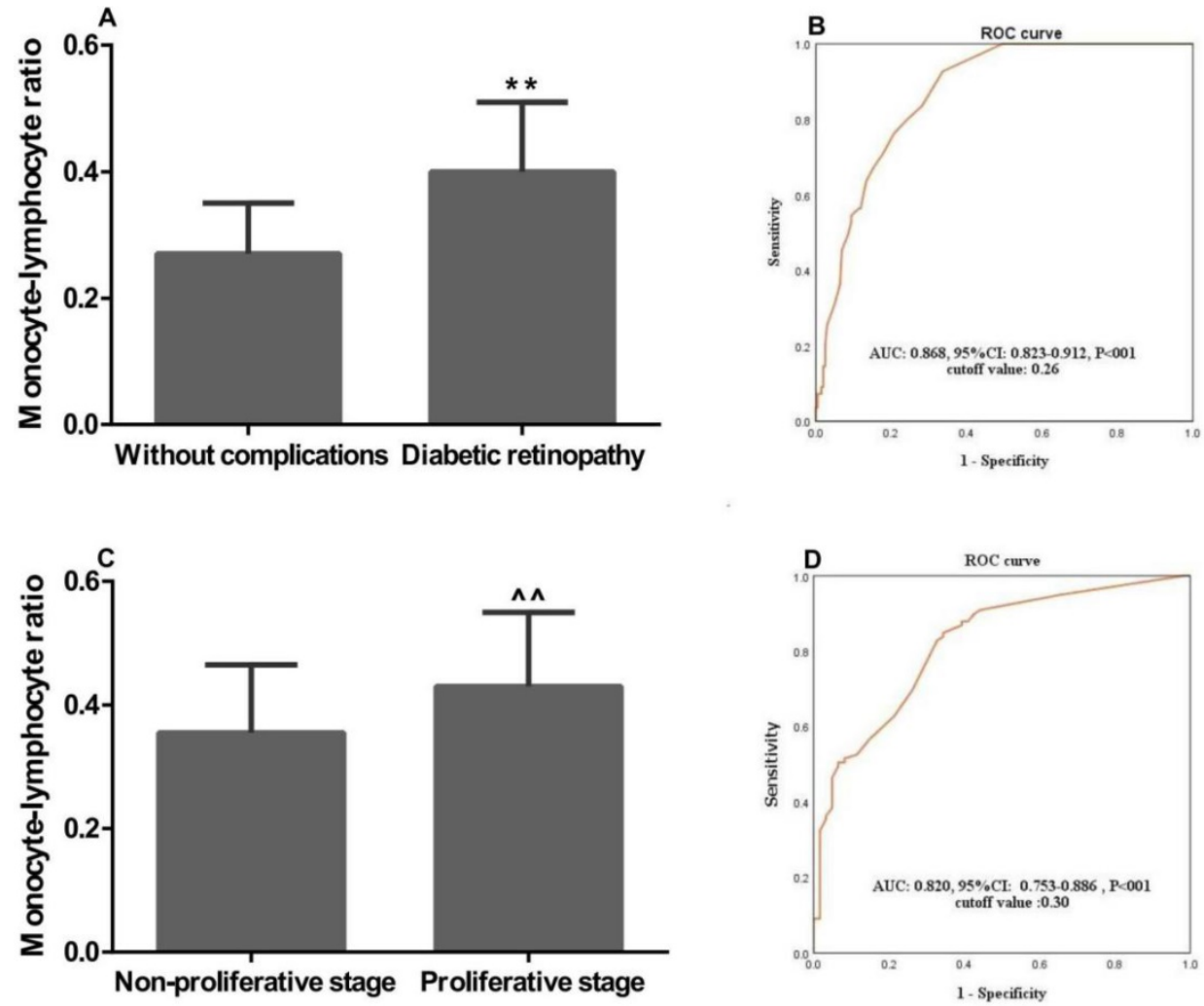

Figure 2. Monocyte-lympocyte ratio and the clinical characteristics of patients with T2D and DR. MLR was significantly associated with (A) diabetic retinopathy, and (C) proliferative stage; ROC curve for discriminating between patients with (B) DR and T2D without complications, and (D) non-proliferative and proliferative stage; ROC: Receiver-operating characteristic; T2D: type 2 diabetes; DR: diabetic retinopathy; AUC: area under the curve. **, ^^, P<0.01 vs. "Without complications" and “Non-proliferative stage" patients by the t-test, respectively.
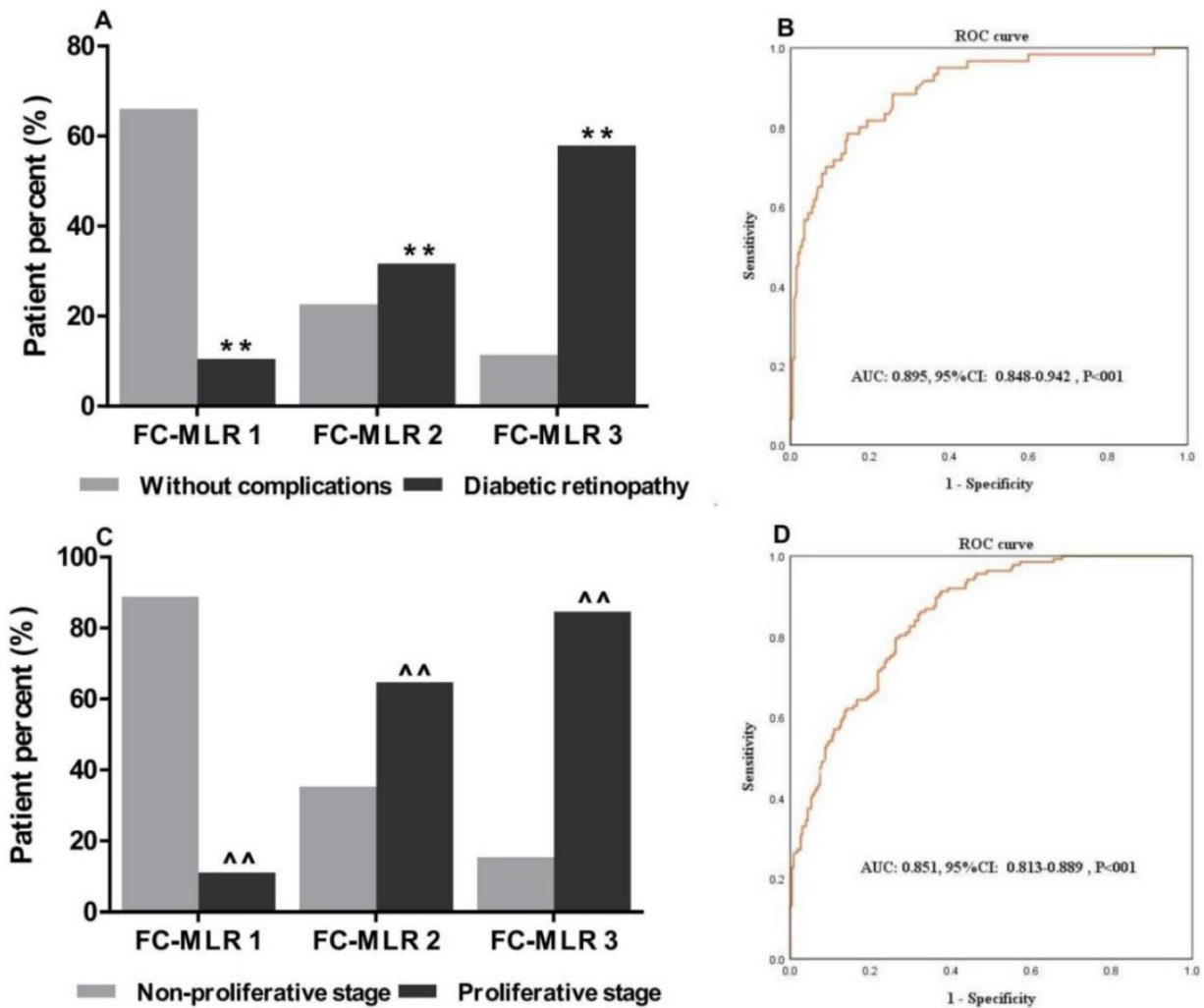

Figure 3. Associations of combined plasma fibrinogen concentrations and MLR with the clinical characteristics of patients with T2D and DR. FC-MLR was significantly associated with (A) diabetic retinopathy, and (C) proliferative stage; ROC curve for discriminating between the patients with (B) DR and T2D without complications, and (D) non-proliferative and proliferative stage; ROC: Receiver-operating characteristic; T2D: type 2 diabetes; DR: diabetic retinopathy; AUC: area under the curve. **, $\wedge \wedge$, $\mathrm{P}<0.01$ vs. "Without complications" and "Non-proliferative stage" patients by the Chi-square test, respectively. 
Fibrinogen is an acute-phase reactant protein that is released in response to infection or systemic inflammation [23]. Moreover, it has been well established that total fibrinogen is elevated during inflammation [24]. Many studies have revealed that patients with diabetic microvascular complications would have high plasma fibrinogen concentrations, and plasma fibrinogen was significantly increased with severity of diabetic retinopathy $[25,26,27]$. In the present study, higher Fbg concentrations were found in DR patient than that without complications, which indicated that the elevation of Fbg level is associated with DR. It has been well known that fibrinogen may induce the synthesis of interleukin-6, an inflammatory mediator, or interact with leukocytes [23]. Therefore, hyperfibrinogenemia may occur secondary to chronic inflammation that occurs in response to the occurrence and progression of DR, which may reversely cause the increasing of plasma Fbg concentrations, and measurement of plasma Fbg will be useful in discriminating patients from DR and T2D without complications. Our study further showed that plasma Fbg had a powerful diagnostic significance with a high AUC and medium sensitivity in discriminating between patients with DR and T2D without complications. Thus, the findings suggest that plasma Fbg also is a useful predictor for the occurrence of DR. The study further showed that there was a higher plasma Fbg concentration in DR patient with proliferative stage than that with non-proliferative stage, and plasma $\mathrm{Fbg}$ also had a powerful diagnostic significance with a high AUC and high sensitivity according to the optimal cutoff value in discriminating between DR patients with proliferative and non-proliferative stage. Our findings revealed that elevated plasma Fbg concentrations were closely associated with high proliferative stage of DR, and Fbg will be a valuable predictor for the progression of DR. Therefore, plasma Fbg can be used as a predictor for the occurrence and progression of DR.

It is known that DR are closely associated with chronic inflammatory responses [28]. A systemic inflammatory response causes variations in the balance of circulating white blood cell constituents [29]. Grossmann et al. showed that WBCs, granulocytes, and monocytes gradually increased from normoglycemic subjects to subjects with diabetes, whereas the lymphocyte concentration was stable despite the disease progression in diabetes [30]. And Luo et al. reported that NLR and PLR could be recommended as diagnostic biomarkers for DR [31]. Moreover, Ji et al. also reported that monocyte to lymphocyte ratio (MLR) or lymphocyte to monocyte ratio (LMR) could mirror the circulating immune status of the host [32]. Thus, these findings suggest that systemic inflammatory response can induce an increased monocyte and decreased lymphocyte in pathogenesis of DR, and lymphopenia and an elevated monocyte count would stand for a microenvironment surrogate marker of the occurrence and progression of DR. Therefore, MLR might be a good reflection of different clinical conditions in T2D and DR patients. In the present study, we found that DR patients had remarkably higher MLR than those without complications, which indicates that a high MLR in DR patient may be resulted from an increased number of monocytes and a decreased number of lymphocytes because of an enhanced inflammatory response and a reduced immune function. Therefore, the findings suggest that MLR is closely associated with the risk of DR. Concerning the increasing of the MLR, we speculate that it may be associated with the production of the pro-inflammatory chemokines such as IL-6, TNF-a, IL-1 $\beta$, and MCP-1 which play major role in the recruitment and activation of monocytes and leukocytes and the subsequent inflammatory responses in DR patients [9]. Thus, observing the changes in monocytes and lymphocytes of peripheral blood is sure to have clinical significance in discriminating patients from DR and T2D without complications. Additionally, our findings also showed that MLR had a high AUC and high sensitivity in discriminating DR and T2D without complications, indicating that MLR is a powerful predictor for the occurrence of DR. And the study further demonstrated a significantly higher MLR in DR patient with proliferative stage than that with non-proliferative stage, and MLR also had a high AUC and high sensitivity in discriminating DR patients between proliferative and non-proliferative stage. Our findings were not consistent with that of the previous study [33], which may be because of regional differences between the included subjects. In the study, our findings reveal a close association of elevated MLR with enhanced severity of DR, indicating that MLR is a powerful predictor for the progression of DR. Therefore, our findings suggest that MLR measurement will be valuable for predicting the occurrence and progression of DR.

The main finding of the study was that combination of plasma FC-MLR was a novel index for evaluating the occurrence and progression of DR. Multivariate regression analyses indicated that plasma Fbg, MLR and FC-MLR were independently associated with the risk and progression of DR, but FC-MLR showed a more powerful clinical significance in discriminating between DR and T2D without complications as well as advanced (proliferative 
stage) and early (non-proliferative stage) DR than single Fbg or MLR. To further assess the predictive power of FC-MLR, the patients with T2D and DR were divided into three groups according to the cutoff values of plasma Fbg concentrations and MLR. We found that patients with both high fibrinogen concentrations and a high MLR had a higher percent of DR patient than did those without high fibrinogen concentrations and a high MLR. And we also found that DR patients with high fibrinogen concentrations and a high MLR were closely associated with a higher patient percent in proliferative stage than did other DR patients. Furthermore, the ROC curve of FC-MLR showed a greater predictive power with high sensitivity and specificity in discriminating between DR and T2D without complications as well as advanced and early DR than did Fbg concentrations or MLR. Therefore, our findings suggest that plasma fibrinogen concentrations combined MLR before treatment would be useful for assessing the risk and progression of DR in patients with T2D. This information can be used to prevent and treat diabetic microvascular complications, which may be practical for the improvement of patient prognosis. FC-MLR is a simple index which is obtained from Fbg concentrations and calculated MLR derived from conventional blood analysis. Consequently, FC-MLR can be used as a cost-effective, convenient, practical, and powerful predictor for DR and a prognostic index in patients with T2D.

There may be some limitations of the present study. In our study, we only reviewed 95 DR patients in one hospital, and there probably were some subclinical DR patients or patients with other complications which were not diagnosed before treatment. On the other hand, the predictive models with the cutoff values and AUCs of ROC curves in the present study did not be externally validated in different study population and other centers. Therefore, the results may have been influenced by the small sample size, the fact that we did not perform different population and multicentric study, and the mismatched patients. However, the present study also reveals a notable significance of combined plasma fibrinogen concentrations and MLR for assessing the occurrence and progression of diabetic retinopathy.

\section{Conclusions}

This study suggests that combined plasma fibrinogen concentrations and MLR is closely associated with the occurrence and severity of diabetic retinopthy and FC-MLR may be a useful predictor for the risk and progression of diabetic retinopthy. However, we should further emphasize that our conclusion could not be used in patients with acute infection because of a close association of MLR with some viral infections.

\section{Abbreviations}

T2D: type 2 diabetes; DR: diabetic retinopathy; FC: fibrinogen concentrations; PDR: proliferative diabetic retinopathy; NPDR: non-proliferative diabetic retinopathy; FPG: fast plasma glucose; PT: prothrombin time; INR: international normalized ratio; aPTT: activated partial thromboplastin time; PLT: platelet count; D-D: D-dimer; TT: thrombin time; WBC: white blood cell; CRP: C-reactive protein; eGFR: estimated glomerular filtration rate; UAE: urinary albumin excretion; ROC: receiver operating characteristic; AUC: area under curve; OR: odds ratio; CI: confidence interval.

\section{Acknowledgements}

The authors thank all those who supported them in patient recruitment and helped gather patient information and perform the statistical analysis.

\section{Funding}

This work was supported by the Public Welfare Technology Research Project of Zhejiang Province, China (Grant number LGD20H070002) granted to Huang QH, and the Medicine and Health Science and Technology Project of Zhejiang Province (Grant number 2020KY022 and 2021KY060) and the Natural Science Foundation of Zhejiang Province (Grant number LY17H080007) granted to FEI XM.

\section{Competing Interests}

The authors have declared that no competing interest exists.

\section{References}

1. Bourne RR, Stevens GA, White RA, Smith JL, Flaxman SR, Price H, et al. Causes of vision loss worldwide, 1990-2010: A systematic analysis. Lancet Glob Health. 2012; 1: 339-349.

2. Sinclair SH, and Schwartz SS. Diabetic Retinopathy-An Underdiagnosed and Undertreated Inflammatory, Neuro-Vascular Complication of Diabetes. Front Endocrinol (Lausanne). 2019; 10: 843.

3. Abbasi A, Peelen LM, Corpeleijn E et al. Prediction models for risk of developing type 2 diabetes: systematic literature search and independent external validation study. BMJ. 2012; 345: e5900.

4. Ho H, Cheung CY, Sabanayagam $\mathrm{C}$, et al. Retinopathy Signs Improved Prediction and Reclassification of Cardiovascular Disease Risk in Diabetes: A prospective cohort study. Sci Rep. 2017; 7: 41492.

5. Abbasi A, Sahlqvist AS, Lotta L, et al. A Systematic Review of Biomarkers and Risk of Incident Type 2 Diabetes: An Overview of Epidemiological, Prediction and Aetiological Research Literature. PLoS One. 2016; 11: e163721.

6. Toth PP, Simko RJ, Palli SR, et al. The impact of serum lipids on risk for microangiopathy in patients with type 2 diabetes mellitus. Cardiovasc Diabetol. 2012; 11: 109 .

7. Chakrabarti S, Khan ZA, Cukiernik $\mathrm{M}$, et al. C-peptide and retinal microangiopathy in diabetes. Exp Diabesity Res. 2004; 5: 91-96.

8. Fickweiler W, Aiello LP, Sun JK, King GL. Retinol binding protein 3 as biomarker for diabetic retinopathy. Ann Transl Med. 2019; 7: 706.

9. Rübsam A, Parikh S, and Fort PE. Role of Inflammation in Diabetic Retinopathy. Int J Mol Sci. 2018; 19: 942.

10. Jenkins AJ, Joglekar MV, Hardikar AA, Keech AC, $\mathrm{O}^{\prime} \mathrm{Neal}$ DN, and Januszewski AS. Biomarkers in Diabetic Retinopathy. Rev Diabet Stud. 2015; 12: 159-195. 
11. Liu J, Liu X, Li Y, et al. The association of neutrophil to lymphocyte ratio, mean platelet volume, and platelet distribution width with diabetic retinopathy and nephropathy: a meta-analysis. Biosci Rep. 2018; 38: BSR20180172.

12. Yuan C, Li N, Mao X, et al. Elevated pretreatment neutrophil/white blood cell ratio and monocyte/lymphocyte ratio predict poor survival in patients with curatively resected non-small cell lung cancer: Results from a large cohort. Thorac Cancer. 2017; 8: 350-358.

13. Xiang F, Chen R, Cao X, et al. Monocyte/lymphocyte ratio as a better predictor of cardiovascular and all-cause mortality in hemodialysis patients: A prospective cohort study. Hemodial Int. 2018; 22: 82-92.

14. Tennent GA, Brennan SO, Stangou AJ, O'Grady J, Hawkins PN and Pepys MB. Human plasma fibrinogen is synthesized in the liver. Blood. 2007; 109: 1971-1974.

15. Weisel JW. Fibrinogen and fibrin. Adv Protein Chem. 2005; 70: 247-299.

16. Pan LL, Ye YL, Wo MY, et al. Clinical Significance of Hemostatic Parameters in the Prediction for Type 2 Diabetes Mellitus and Diabetic Nephropathy. Dis Markers. 2018; 2018: 5214376

17. Cao YY, Ni XW, Wang $Y$, et al. Clinical and prognostic significance of combined plasma fibrinogen concentrations and the monocyte-to-lymphocyte ratio in patients with ovarian cancer. Ann Transl Med. 2019; 7: 242.

18. China Diabetes Association. China Diabetes prevention and Treatment Guideline 2013 (In Chinese). Chin J Diabete. 2014; 22:2-42.

19. Wang W and Lo Amy CY. Diabetic Retinopathy: Pathophysiology and Treatments. Int J Mol Sci. 2018; 19: 1816.

20. Fei X, Xing M, Wo M, et al. Thyroid stimulating hormone and free triiodothyronine are valuable predictors for diabetic nephropathy in patient with type 2 diabetes. Ann Trans Med. 2018; 6: 305.

21. Fei X, Wang H, Yuan W, Wo M, Jiang L. Tissue Factor Pathway Inhibitor-1 Is a Valuable Marker for the Prediction of Deep Venous Thrombosis and Tumor Metastasis in Patients with Lung Cancer. Biomed Res Int. 2017; 2017: 8983763.

22. Chi XH, Li GP, Wang QS, et al. CKD-EPI creatinine-cystatin C glomerular filtration rate estimation equation seems more suitable for Chinese patients with chronic kidney disease than other equations. BMC Nephrol. 2017; 18: 226.

23. Zhang $X$, Long Q. Elevated serum plasma fibrinogen is associated with advanced tumor stage and poor survival in hepatocellular carcinoma patients. Medicine (Baltimore). 2017; 96: e6694.

24. Alexander KS, Madden TE, and Farrell DH. Association Between $\gamma^{\prime}$ Fibrinogen Levels and Inflammation. Thromb Haemost. 2011; 105: 605-609.

25. Le DS, Miles R, Savage PJ, et al. The association of plasma fibrinogen concentration with diabetic microvascular complications in young adults with early-onset of type 2 diabetes. Diabetes Res Clin Pract. 2008; 82: 317-323.

26. Goldberg RB. Cytokine and cytokine-like inflammation markers, endothelial dysfunction, and imbalanced coagulation in development of diabetes and its complications. J Clin Endocrinol Metab. 2009; 94: 3171-3182.

27. Zheng N, Shi X, Chen X, et al. Associations between Inflammatory Markers, Hemostatic Markers and Microvascular Complications in 182 Chinese Patients with Type 2 Diabetes Mellitus. Lab Med. 2015; 46: 214-220.

28. Semeraro F, Cancarini A, dell'Omo R, Rezzola S, Romano MR, and Costagliola C. Diabetic Retinopathy: Vascular and Inflammatory Disease. J Diabetes Res. 2015; 2015: 582060

29. Satomi A, Murakami S, Ishida K, Mastuki M, Hashimoto T, Sonoda M. Significance of increased neutrophils in patients with advanced colorectal cancer. Acta Oncol. 1995; 34: 69-73.

30. Grossmann V, Schmitt VH, Zeller T, Panova-Noeva M, Schulz A, Laubert-Reh $\mathrm{D}$, et al. Profile of the Immune and Inflammatory Response in Individuals With Prediabetes and Type 2 Diabetes. Diabetes Care. 2015; 38: 1356-1364.

31. Luo WJ and Zhang WF. The relationship of blood cell-associated inflammatory indices and diabetic retinopathy: a Meta-analysis and systematic review. Int J Ophthalmol. 2019; 12: 312-323.

32. Ji H, Niu X, Yin L, Wang Y, Huang L, Xuan Q, et al. Ratio of Immune Response to Tumor Burden Predicts Survival Via Regulating Functions of Lymphocytes and Monocytes in Diffuse Large B-Cell Lymphoma. Cell Physiol Biochem. 2015; 45: 951-961.

33. Yue $\mathrm{S}$, Zhang $\mathrm{J}, \mathrm{Wu}$ J, et al. Use of the monocyte-to-lymphocyte ratio to predict diabetic retinopathy. Int J Environ Res Public Health. 2015; 12: 10009-10019. 